ISSN-L 2077-0014

DOI: https://doi.org/10.33326/26176068.2020.2.1015

Reporte de caso/Case report/Relatório do caso

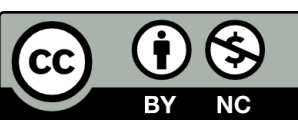

\title{
Convulsiones asociadas con clozapina
}

\author{
Seizure Associated With Clozapine \\ Convulsões associadas à clozapina
}

Joshep Revilla-Zúñiga ${ }^{1,2, a}$

(iD) https://orcid.org/0000-0003-3224-3763

José Revilla-Urquizo $3,4,5, a$

(iD https://orcid.org/0000-0002-5961-2760

Carolina Dávalos-Luque ${ }^{4, b}$

(iD) https://orcid.org/0000-0002-5586-0919

\section{Resumen}

Objetivo: Los antipsicóticos son medicamentos que pueden disminuir el umbral convulsivo. La clozapina es un antipsicótico atípico usado en el tratamiento de la esquizofrenia resistente a los antipsicóticos convencionales y está asociado a convulsiones como efecto adverso. Caso clínico: Varón de 28 años con diagnóstico de esquizofrenia paranoide que ingresó a un servicio de psiquiatría de un hospital general por síntomas psicóticos de difícil manejo. Por la persistencia de los síntomas psicóticos a pesar del uso de medicación antipsicótica, y la ideación suicida, se decidió el inicio de clozapina. Presentó dos crisis convulsivas, la primera con $700 \mathrm{mg} / \mathrm{d}$ de clozapina y la segunda con $600 \mathrm{mg} / \mathrm{d}$, que remitieron tras la suspensión de este antipsicótico. Debido a este evento adverso, no se reinició la clozapina y se inició la terapia electroconvulsiva, con una respuesta clínica favorable. Resultados: La clozapina es uno de los antipsicóticos que más se asocia a la disminución del umbral convulsivo y que no contraindica su uso. Conclusiones: Si bien en el caso presentado se suspendió la clozapina por la aparición de dos eventos convulsivos, y se decidió el uso de la terapia electroconvulsiva con una respuesta clínica favorable, existen otras estrategias que pueden utilizarse para el manejo de este efecto adverso.

Palabras clave: antipsicóticos, convulsiones epilépticas, crisis convulsivas, evento adverso

\section{Abstract}

Objective: Antipsychotics are medications that can lower the seizure threshold. Clozapine is an atypical antipsychotic used in treatment resistant schizophrenia to conventional antipsychotics and it is associated with seizures as an adverse event. Clinical case: A 28-year-old man with a diagnosis of paranoid schizophrenia who was admitted to a psychiatric service of a general hospital due to difficult-to-manage psychotic symptoms. Due to the persistence of psychotic symptoms, despite the use of antipsychotic medication and suicidal ideation, it was decided to start clozapine. He had two seizures, the first with $700 \mathrm{mg} / \mathrm{d}$ of clozapine and the second with $600 \mathrm{mg} / \mathrm{d}$, which remitted after the suspension of this antipsychotic. Due to this adverse event, clozapine was not restarted and electroconvulsive therapy was started, with a favorable clinical response. Results: Clozapine is one of the antipsychotics that is most associated with the reduction of the seizure threshold and that does not contraindicate its use. Conclusions: Although clozapine was suspended due to the appearance of two seizure events, and the use of electroconvulsive therapy was decided with a favorable clinical response, other strategies can be used to manage this adverse effect.

Keywords: epileptic seizures, convulsive seizures, antipsychotic agents, adverse event.

\footnotetext{
${ }^{1}$ Facultad de Medicina Alberto Hurtado, Universidad Peruana Cayetano Heredia. Lima, Perú

${ }^{2}$ Instituto Nacional de Salud Mental "Honorio Delgado- Hideyo Noguchi". Lima, Perú

${ }^{3}$ Facultad de Ciencias de la Salud, Universidad Nacional Jorge Basadre Grohmann. Tacna, Perú

${ }^{4}$ Facultad de Ciencias de la Salud, Universidad Privada de Tacna. Tacna, Perú

${ }^{5}$ Hospital Regional Hipólito Unanue. Tacna, Perú

${ }^{\text {a }}$ Médico-Psiquiatra

${ }^{\mathrm{b}}$ Médico Residente de Psiquiatría
} 


\section{Resumo}

Objetivo: Os antipsicóticos são medicamentos que podem diminuir o limiar convulsivo. A clozapina é um antipsicótico atípico usado no tratamento da esquizofrenia resistente aos antipsicóticos convencionais e está associada a convulsões como efeito adverso. Caso clínico: Homem de 28 anos com diagnóstico de esquizofrenia paranóide que deu entrada no serviço psiquiátrico de um hospital geral devido a sintomas psicóticos de difícil tratamento. Devido à persistência dos sintomas psicóticos apesar do uso de medicação antipsicótica, e ideação suicida, optou-se pelo início da clozapina. Ele teve duas convulsões, a primeira com $700 \mathrm{mg} / \mathrm{d}$ de clozapina e a segunda com $600 \mathrm{mg} / \mathrm{d}$, que remitiram após a suspensão desse antipsicótico. Devido a esse evento adverso, a clozapina não foi reiniciada e a eletroconvulsoterapia foi iniciada, com resposta clínica favorável. Resultados: A clozapina é um dos antipsicóticos que mais está associado à redução do limiar convulsivo e que não contra-indica seu uso. Conclusões: Embora no caso apresentado a clozapina tenha sido suspensa devido ao aparecimento de dois episódios convulsivos, e o uso da eletroconvulsoterapia tenha sido decidido com resposta clínica favorável, existem outras estratégias que podem ser utilizadas para o manejo desse efeito adverso.

Palavras-chave: antipsicóticos, convulsões epilépticas, convulsões, evento adverso

\section{Introducción}

La clozapina es un antipsicótico atípico aprobado para la esquizofrenia resistente al tratamiento. ${ }^{1}$ Una de las principales razones para reservar su uso en estos casos es el riesgo de agranulocitosis (conteo absoluto de neutrófilos < 500 por microlitro), evento que tiene una prevalencia del $0,9 \%{ }^{2}$

En general, todos los antipsicóticos pueden disminuir el umbral convulsivo y generar una convulsión, sin embargo, la clozapina posee un mayor riesgo, aproximadamente entre 1-6\%, siendo mayor si la dosis es superior a $600 \mathrm{mg} / \mathrm{d}^{3}$

La terapia electroconvulsiva (TEC) es un tratamiento médico por medio del cual se administra un estímulo eléctrico de frecuencia variable al cerebro a través del cuero cabelludo para la inducción de una convulsión terapéutica. En la revisión Cochrane del 2019, se concluyó que la TEC tiene un efecto positivo a mediano plazo en la respuesta clínica de los pacientes con esquizofrenia resistente al tratamiento. ${ }^{4}$

A continuación, presentamos el caso de un varón con diagnóstico de esquizofrenia paranoide que ingresó a un hospital general por síntomas psicóticos, y que tras no haber respuesta con dos antipsicóticos previos y la aparición de ideación suicida se decidió el uso de clozapina, presentando posteriormente dos episodios de convulsiones tónico-clónicas generalizadas. Por este motivo, se decidió la suspensión de la clozapina y el inicio de la TEC, con respuesta favorable.

\section{Reporte de caso}

Es un varón de 28 años, con el diagnóstico de esquizofrenia paranoide desde hace 8 años, con un tratamiento irregular, quien ingresó al Servicio de Psiquiatría del Departamento de Salud Mental del Hospital Hipólito Unanue de Tacna por conducta agresiva hacia la familia no controlable en casa. Estos síntomas también consistieron en alucinaciones auditivas, delusiones de daño, delusiones místicas, ideas de culpa, irritabilidad y conducta desorganizada.

Se inició el haloperidol a dosis de 30 $\mathrm{mg} /$ día durante 20 días, logrando disminuir la agresividad, pero se mantenían el resto de síntomas psicóticos, sin mejoría clínica significativa. Se continuó el tratamiento con risperidona, llegando a una dosis máxima de 10 $\mathrm{mg} /$ día por un período de 51 días, durante los cuales se evidenció leve mejoría, con 
disminución de las alucinaciones auditivas y las delusiones de daño, pero se habían agregado ideas de suicidio, incremento de las delusiones místicas y ansiedad. Por la ideación suicida y la escasa mejoría clínica, se decidió iniciar clozapina, con una dosis inicial de 200 mg/día, con incrementos progresivos hasta una dosis de $700 \mathrm{mg} / \mathrm{día}$ en un lapso de 36 días. A los 6 días de esta dosis de clozapina, el paciente presentó una convulsión tónico-clónica generalizada, sin relajación de esfínteres, por lo que se disminuyó la dosis a $500 \mathrm{mg} / \mathrm{día}$, sin reaparición de este evento. Tras 10 días con dicha dosis, se decidió incrementar la medicación a $600 \mathrm{mg} /$ día por la persistencia de la sintomatología psicótica, produciéndose un nuevo episodio convulsivo, con características similares al anterior. Por este motivo, se disminuyó a $500 \mathrm{mg} / \mathrm{día}$. Con esta dosis, no hubo nuevos eventos convulsivos. Como no había tolerancia al aumento de la clozapina por los eventos convulsivos y por la persistencia de síntomas psicóticos e ideación suicida, se decidió suspender la medicación e iniciar Terapia Electroconvulsiva, con tres sesiones interdiarias por el lapso de dos semanas (6 sesiones en total), reduciendo en su totalidad la sintomatología psicótica y la ideación suicida. Luego de estas sesiones, recibió dos sesiones más (con diferencia de un día) a los 15 días de la sexta. A los 20 días de la última sesión de TEC, se repitieron 2 sesiones más de TEC (con diferencia de un día). Luego se volvió a administrar 2 sesiones de TEC a los 30 días. Continuó con esta frecuencia de 2 sesiones de TEC cada 30 días por 4 meses más, y por la mejoría clínica es dado de alta con la indicación de 2 sesiones de TEC cada 30 días de forma ambulatoria. No recibió algún psicofármaco y no volvió a presentar otro evento convulsivo. Desde entonces ha mantenido su mejoría clínica sin psicofármacos y solo con 2 sesiones de TEC cada 30 días.

El paciente no tenía historia de convulsiones previas y no había historia de epilepsia en la familia. No tenía hábitos nocivos de consumo de alcohol, tabaco o de sustancias psicoactivas, ni antecedentes de traumatismos craneales previos.
Se realizaron controles analíticos periódicos, con lectura de hemograma dentro de parámetros normales antes y durante el tratamiento con clozapina.

El perfil hepático, lipídico, glucosa y creatinina se encontraban dentro de parámetros normales en todos los controles realizados. Se realizó una tomografía espiral multicorte cerebral sin contraste, que no reportó anormalidad y el electroencefalograma estaba dentro de parámetros normales.

\section{Discusión}

Las convulsiones por la clozapina tienen un mecanismo de inducción no del todo dilucidado. Se postula que el bloqueo de los receptores de dopamina D4 a nivel mesolímbico, en donde las estructuras que lo conforman están relacionadas a áreas de inicio de convulsiones, puede explicar su alta capacidad epileptogénica en comparación con otros antipsicóticos. ${ }^{5,6}$ Otros posibles mecanismos son el efecto que puede tener sobre los receptores de ácido gammaaminobutírico $A$, de la acetilcolina tipo nicotínico, del glutamato tipo N-metil-Daspartato, de serotonina $5-\mathrm{HT} 2 \mathrm{~A}$, y de glicina sensible-estricnina. ${ }^{\top}$

Los tipos de convulsiones secundarias a la clozapina pueden ser tónico-clónica generalizada, mioclónica/atónica generalizada, parcial compleja y simple, y por último la de ausencia. El tipo de convulsión más frecuente es la tónico-clónica generalizada, con una incidencia que oscila entre el 1,3\% hasta el 6 $\%{ }^{8}$ A menudo, la aparición de ataques mioclónicos o "tirones mioclónicos", usualmente en región orofacial, incrementan el riesgo de una convulsión tónico-clonica generalizada secundaria o una convulsión mioclónica. ${ }^{9}$

Sobre el riesgo de las convulsiones por clozapina, es mayor con el aumento de la dosis, encontrándose entre $0,6 \%$ - $2 \%$ si la dosis es menor a $300 \mathrm{mg} / \mathrm{d}$, de 1,8 $\%$ - $4 \%$ si la dosis está entre $300-599 \mathrm{mg} / \mathrm{d}$, y de $5 \%$ - $14 \%$ si la 
dosis se encuentra entre $600-900 \mathrm{mg} / \mathrm{d}^{7}$ Sin embargo, estos eventos también pueden ocurrir con dosis menores de clozapina, por lo cual la exploración de factores de riesgo adicionales debe ser la conducta a seguir. ${ }^{8}$

Algunos de los factores de riesgo identificados para las convulsiones por clozapina son el incremento de la dosis rápidamente, las dosis altas, la historia de episodios convulsivos o anormalidades en el EEG previas, trastornos del sistema nervioso central, historia de traumas craneales, uso concomitante de medicamentos epileptogénicos, abuso de alcohol e historia familiar de epilepsia. ${ }^{10}$ Las concentraciones plasmáticas de clozapina mayor a $1000 \mathrm{ng} / \mathrm{mL}$ $(3 \mu \mathrm{mol} / \mathrm{L})$ también están asociadas a un mayor riesgo de convulsiones. ${ }^{11}$

Es importante también recordar que la vía de metabolización preferente de la clozapina es la isoenzima $1 \mathrm{~A} 2$ del citocromo P450 (CYP), por lo tanto, se debe valorar los medicamentos y drogas que puedan ser o inhibidores de esta vía, como la fluvoxamina y el ciprofloxacino, que incrementarían las concentraciones séricas, o inductores, como el tabaco, que disminuirían las concentraciones de clozapina. ${ }^{8}$

La clozapina tiene un mayor riesgo de anormalidades en el electroencefalograma (EEG) en comparación con el resto de antipsicóticos, ${ }^{12}$ sin embargo, no se ha establecido que sea un predictor de riesgo de convulsión. ${ }^{9}$

La presentación de un episodio convulsivo con el uso de clozapina no es una contraindicación para el tratamiento con este antipsicótico. Para estos casos, se recomienda suspender la clozapina por 24 horas, luego reiniciar la medicación a una dosis menor (mitad de la dosis) con la inclusión de un medicamento anticonvulsivante..$^{13}$ Dentro de los medicamentos anticonvulsivantes que pueden utilizarse se encuentran el ácido valproico, la gabapentina, la lamotrigina, el topiramato, la fenitoína, el fenobarbital y el clonazepam. No se recomienda el uso de carbamazepina con clozapina por el mayor riesgo de neutropenia y la interacción farmacológica que disminuye en un $50 \%$ las concentraciones de clozapina. ${ }^{8} \mathrm{El}$ más usado de los medicamentos anticonvulsivantes es el ácido valproico por sus efectos como estabilizador del ánimo, un mínimo efecto en el metabolismo de la clozapina y una reducción en el patrón de poliespiga y onda generalizadas en los pacientes que están en tratamiento con clozapina. ${ }^{14}$ Es también importante una evaluación médica completa para descartar otras posibles causas de la convulsión en los pacientes en tratamiento con clozapina.

Sobre la TEC en la esquizofrenia, hay evidencia de su utilidad en pacientes con resistencia al tratamiento, en condiciones que necesitan una mejoría global rápida, como son la conducta agresiva hacia los demás, la conducta suicida y los síntomas catatónicos. ${ }^{15}$

En relación a nuestro caso, el diagnóstico de convulsiones inducidas por la clozapina se formuló en base a la ausencia de factores de riesgo para las convulsiones y la exclusión de otras posibles causas con exámenes auxiliares como la tomografía espiral multicorte cerebral y el electroencefalograma. El paciente tuvo 2 episodios convulsivos por las dosis altas de la clozapina, por lo que se decidió la suspensión de la medicación y el uso de la TEC, con una respuesta favorable sobre los síntomas psicóticos y la ideación suicida.

En conclusión, las convulsiones asociadas a clozapina son un efecto adverso que amerita la exploración de varios factores de riesgo, y que no contraindica el uso de este antipsicótico reservado para casos de esquizofrenia resistente al tratamiento, entre otros. Si bien en la práctica clínica se considera que dosis mayores a $600 \mathrm{mg} / \mathrm{d}$ son un factor de riesgo para convulsiones, la literatura científica demuestra que también puede producirse a menores dosis, por lo que el uso profiláctico de un anticonvulsivante es discutible. 


\section{Referencias}

1. Warnez S, Alessi-Severini S. Clozapine: A review of clinical practice guidelines and prescribing trends. BMC Psychiatry. $\begin{array}{lllllllllll}2 & 0 & 1 & 4 & ; & 1 & 4 & : & 1 & 0 & 2\end{array}$. http://dx.doi.org/10.1186/1471-244X-14-102

2. Legge SE, Walters JT. Genetics of clozapineassociated neutropenia: Recent advances, challenges and future perspective. Pharmacogenomics. 2019;20(4):279-90. http://dx.doi.org/10.2217/pgs-2018-0188

3. De Berardis D, Rapini G, Olivieri L, di Nicola D, Tomasetti C, Valchera A, et al. Safety of antipsychotics for the treatment of schizophrenia: a focus on the adverse effects of clozapine. Ther Adv Drug Saf. $2018 ; 9(5): 237-56$. http://dx.doi.org/10.1177/204209861875626 1

4. Sinclair DJM, Zhao S, Qi F, Nyakyoma K, Kwong JSW, Adams CE. Electroconvulsive therapy for treatment-resistant schizophrenia. Cochrane Database Syst R e v . 2019 ; 3 : C D 011847 . http://dx.doi.org/10.1002/14651858.CD0118 47.pub2

5. Pisani F, Oteri G, Costa C, di Raimondo G, di Perri R. Effects of psychotropic drugs on seizure threshold. Drug Saf. 2002; $25(2): 91-\begin{array}{llll}1 & 1 & 0\end{array}$. http://dx.doi.org/10.2165/00002018200225020-00004

6. Mansour A, Meador-Woodruff J, Burke S, Bunzow J, Akil H, Van Tol HHM, Civelli, O.; and Watson S. J. Differential distribution of D2 and D4 dopamine receptor mRNAs in rat brain: An in situ hybridization study. Soc Neurosci Abstr. 1991;17:599.

7. Kikuchi YS, Sato W, Ataka K, Yagisawa K, Omori Y, Kanbayashi T., Shimizu T. Clozapine-induced seizures, electroencephalography abnormalities, and clinical responses in Japanese patients with schizophrenia. Neuropsychiatr Dis Treat. $\begin{array}{llllllllllllll}2 & 0 & 1 & 4 & ; & 1 & 0 & : & 1 & 9 & 7 & 3 & - & 8\end{array}$. http://dx.doi.org/10.2147/NDT.S69784

\section{Correspondencia:}

joshep.revilla.z@upch.pe
8. Williams AM, Park SH. Seizure associated with clozapine: Incidence, etiology, and management. CNS Drugs. 2015; $29(2): 1001-11$. http://dx.doi.org/10.1007/s40263-014-0222$y$

9. Wong J, Delva N. Clozapine-induced seizures: Recognition and treatment. Can J Psychiatry. 2007;52(7):457-63. http://dx.doi.org/10.1177/070674370705200 708

10. Bolu A, Akarsu S, Pan E, Aydemir E, Oznur T. Low-dose Clozapine-induced Seizure: A Case Report. Clin Psychopharmacol Neurosci. $2017 ; 15(2): 190-3$. http://dx.doi.org/10.9758/cpn.2017.15.2.190

11. Freeman DJ, Oyewumi LK. Will routine therapeutic drug monitoring have a place in clozapine therapy? Clin Pharmacokinet. 1997 ; $32(2)$ : $93-100$. http://dx.doi.org/10.2165/00003088199732020-00001

12. Centorrino F, Price BH, Tuttle M, Bahk WM, Hennen J, Albert MJ, Baldessarini, R.J. EEG abnormalities during treatment with typical and atypical antipsychotics. Am J Psychiatry. $2002 ; 159(1): 109-15$. http://dx.doi.org/10.1176/appi.ajp.159.1.109

13. Foster R, Olajide D. A case of clozapineinduced tonic-clonic seizures managed with valproate: Implications for clinical care. J Psychopharmacol. 2005;19(1):93-6. http://dx.doi.org/10.1177/026988110504890 2

14. Grover S, Hazari N, Chakrabarti S, Avasthi A. Association of clozapine with seizures: A brief report involving 222 patients prescribed clozapine. East Asian Arch Psychiatry. $2015 ; 25(2): 73-8$. https://www.ncbi.nlm.nih.gov/pubmed/26118 746

15. Pompili $M$, Lester D, Dominici G, Longo L, Marconi G, Forte A, Serafini G, Amore M, Girardia P.Indications for electroconvulsive treatment in schizophrenia: A systematic review. Schizophr Res. 2013;146(1-3):1-9. http://dx.doi.org/10.1016/j.schres.2013.02.0 05

Fecha de recepción: 02 de septiembre 2020 Fecha de aceptación: 18 de diciembre 2020 